\title{
Perioperative Medical Complications after Posterior Approach Spinal Instrumentation Surgery for Osteoporotic Vertebral Collapse: A Comparative Study in Patients with Primary Osteoporosis and Those with Secondary Osteoporosis
}

\author{
Naohisa Miyakoshi ${ }^{1,2}$, Takashi Kobayashi ${ }^{2,3}$, Tetsuya Suzuki ${ }^{2,4}$, \\ Kazuma Kikuchi $^{2,3}$, Yuji Kasukawa ${ }^{1,2}$, Yoichi Shimada ${ }^{1,2}$ \\ ${ }^{1}$ Department of Orthopedic Surgery, Akita University Graduate School of Medicine, Akita, Japan \\ ${ }^{2}$ Akita Spine Group (ASG), Akita, Japan \\ ${ }^{3}$ Department of Orthopedic Surgery, Akita Kosei Medical Center, Akita, Japan \\ ${ }^{4}$ Department of Orthopedic Surgery, Akita Red Cross Hospital, Akita, Japan
}

\section{Study Design: A retrospective comparative study.}

Purpose: To compare perioperative medical complications after posterior approach spinal instrumentation surgery for osteoporotic vertebral collapse $(\mathrm{OVC})$ between patients with primary osteoporosis and those with secondary osteoporosis.

Overview of Literature: With increased aging of society, the demand for instrumentation surgery for an osteoporotic spine has been increasing. However, no studies have compared the rates or severities of perioperative complications after spinal instrumentation surgery between patients with primary osteoporosis and those with secondary osteoporosis.

Methods: Ninety-one patients with OVC aged $\geq 50$ years (23 males and 68 females) who underwent posterior approach vertebral replacement with cages or posterior spinal fusion combined with vertebroplasty were divided into primary $(n=56)$ and secondary $(n=35)$ osteoporosis groups. Bone mineral density (BMD), osteoporosis treatment prior to OVC, operative invasiveness, and perioperative medical complications were compared.

Results: Diabetes mellitus (51.4\%) was the most common cause of secondary osteoporosis, followed by glucocorticoid use (22.9\%). No significant differences were seen in terms of age, gender, BMD, osteoporosis treatment, or operative invasiveness, including the number of levels fused, estimated blood loss, and number of patients requiring transfusion. No significant difference in the incidence of perioperative complications were observed between the primary and secondary osteoporosis groups (16.1\% vs. 22.9\%). However, surgical site infection (SSI) was significantly more frequently seen in the secondary osteoporosis group (11.4\%) than in the primary osteoporosis group $(1.8 \% ; p<0.05)$. One patient in the secondary osteoporosis group developed methicillin-resistant Staphylococcus aureus infection that ultimately required instrument removal.

Conclusions: The overall incidence of perioperative medical complications after posterior approach spinal instrumentation surgery for OVC was comparable between the primary and secondary osteoporosis groups under conditions of similar background characteristics and operative invasiveness. However, SSI (particularly more severe cases) occurred more frequently in patients with secondary osteoporosis.

Keywords: Osteoporosis; Vertebral fracture; Complications; Surgical wound infection

Received Dec 13, 2016; Revised Feb 21, 2017; Accepted Mar 19, 2017

Corresponding author: Naohisa Miyakoshi

Department of Orthopedic Surgery, Akita University Graduate School of Medicine, 1-1-1 Hondo, Akita 010-8543, Japan

Tel: +81-18-884-6148, Fax: +81-18-836-2617, E-mail: miyakosh@doc.med.akita-u.ac.jp 


\section{Introduction}

Osteoporosis generally increases bone fragility and leads to an increased risk of fractures, with older women the most severely affected. Vertebral fractures are the most common clinical manifestation of the disease; however, most osteoporotic vertebral fractures respond well to nonsurgical management [1]. However, vertebral fractures may fail to heal in some cases, resulting in progressive collapse and/or pseudoarthrosis. This pathology has been defined as osteoporotic vertebral collapse (OVC) $[2,3]$. Symptomatic OVC often requires surgery due to intolerable pain, progressive spinal kyphosis, and/or neurologic symptoms including paraparesis. With increased aging of society, the demand for instrumentation surgery for a symptomatic osteoporotic spine has been increasing $[1,4]$.

In elderly patients undergoing surgical treatment for OVC with decompression and/or fusion with instrumentation, the presence of multiple comorbid conditions is not uncommon, particularly among patients with secondary osteoporosis. Although proper planning and patient preparation can optimize the medical condition of patients and help decrease the perioperative risk [5], primary diseases or conditions associated with secondary osteoporosis, including diabetes mellitus, chronic kidney disease, and glucocorticoid use, may increase the perioperative risk.

However, to the best of our knowledge, no studies have aimed to compare the rate or severity of perioperative complications after spinal instrumentation surgery between patients with primary osteoporosis and those with secondary osteoporosis. Therefore, the present retrospective study aimed to investigate whether perioperative complications after posterior approach spinal instrumentation surgery for OVC are more frequent in patients with secondary osteoporosis than in those with primary osteoporosis.

\section{Materials and Methods}

\section{Patients and data collection}

Subjects comprised 91 consecutive patients with osteoporosis aged $\geq 50$ years ( 23 males and 68 females) who underwent posterior approach spinal instrumentation surgery for OVC between 2009 and 2014. Osteoporosis was diagnosed according to the criteria proposed by the Japanese Society for Bone and Mineral Research, as de- scribed in the Japanese 2011 guidelines for the prevention and treatment of osteoporosis [6]. Briefly, osteoporosis was diagnosed in patients with (1) any fragility fracture, or (2) bone mineral density (BMD) of less than $70 \%$ of the young adult mean.

Patients were divided into a primary $(\mathrm{n}=56)$ and secondary ( $n=35)$ osteoporosis group according to the causative disease or condition underlying osteoporosis. According to the Japanese 2011 guidelines for the prevention and treatment of osteoporosis [6], secondary osteoporosis is defined as decreased BMD and decreased bone quality due to one or more causes in addition to genetic factors, lifestyle, menopause, and aging. Secondary osteoporosis is caused by other diseases (e.g., hyperparathyroidism, rheumatoid arthritis, etc.), lifestyle-related diseases influencing bone metabolism (e.g., diabetes mellitus, chronic kidney disease, etc.), and treatments for other diseases (e.g., glucocorticoid use, sex hormone-lowering therapy, etc.) [6]. Thus, in the present study, secondary osteoporosis was diagnosed in patients with any of the aforementioned direct causes of osteoporosis (i.e., diabetes mellitus, chronic kidney disease, glucocorticoid use, etc.). Primary osteoporosis was diagnosed in patients with no apparent causes other than aging and/or menopause. Background data including BMD, osteoporosis treatment, operative invasiveness, and perioperative medical complications were reviewed and compared between the groups.

$\mathrm{BMD}$ of the proximal femur was measured using dualenergy X-ray absorptiometry (QDR 4500A, Hologic, Waltham, MA, USA) before surgery. As BMD of the lumbar spine in this population is highly influenced by spinal degeneration and vertebral fractures, BMD data of the lumbar spine were not used. In all patients, surgical procedures, number of levels fused, operation time, and intraoperative estimated blood loss were reviewed as parameters indicating operative invasiveness. Perioperative medical complications were defined as pathological processes that affected patients and occurred during or around the time of surgery (from immediately before surgery to 3 weeks after surgery) that were not directly related to the surgical technique [7]. The present study retrospectively investigated all perioperative medical conditions based on the medical records of each patient, and medical complications were defined as harmful or potentially harmful conditions that required any form of treatment. Deep surgical site infection (SSI) was defined as infection involving the deep muscles or fascia [8]. In 
Table 1. Background data of patients with primary and secondary osteoporosis

\begin{tabular}{lccc} 
Variables & Primary osteoporosis $(\mathrm{n}=56)$ & Secondary osteoporosis $(\mathrm{n}=35)$ & $p$-value \\
Age $(\mathrm{yr})$ & $74.2 \pm 6.8$ & $72.7 \pm 8.1$ & 0.330 \\
Sex (male:female) & $11: 45$ & $12: 23$ & 0.118 \\
\hline BMD of proximal femur $\left(\mathrm{g} / \mathrm{cm}^{2}\right)$ & $0.644 \pm 0.089$ & $0.683 \pm 0.091$ & 0.341 \\
\hline Osteoporosis treatment (yes:no) & $35: 21$ & $19: 16$ & 0.438 \\
\hline
\end{tabular}

Values are presented as mean \pm standard deviation or number of patients. $\mathrm{BMD}$, bone mineral density.

contrast, superficial infection was defined as infection involving the skin or subcutaneous tissue only [8].

Informed consent to the use of their data was obtained from all patients. All procedures in the present study were performed in accordance with the ethical standards of the institutional and/or national research committee and with the 1964 Helsinki Declaration and its later amendments or comparable ethical standards.

\section{Surgical procedure}

Surgical indications for OVC in the present study were weakness (paralysis) of the lower extremities and/or intolerable back pain due to intravertebral instability resulting in an unstable vacuum cleft or increased spinal kyphosis. Indicated patients underwent one of the two following posterior approach spinal instrumentation surgeries. If the fractured vertebra was severely collapsed and showed decreased intravertebral instability, the vertebrae were partially or totally resected using a posterior approach and replaced with large, rectangular, parallelepiped cages and autograft bone and pedicle screwing was then applied 1-3 levels above and below the lesion, considering the affected vertebral level and osteoporosis severity [9]. This technique was termed "posterior approach vertebral replacement with rectangular parallelepiped cage" (PAVREC) [9]. The details of the PAVREC procedure have been described elsewhere [9]. If the fractured vertebra had apparent intravertebral instability, posterior decompression (laminectomy) and vertebroplasty (VP) were applied with hydroxyapatite blocks in combination with instrumented posterior spinal fusion (PSF) 1-3 levels above and below the lesion (VP+PSF).

\section{Statistical analysis}

All data were analyzed using StatView statistical software
Table 2. Use of anti-osteoporosis drugs in both groups

\begin{tabular}{lcc} 
Agents & $\begin{array}{c}\text { Primary } \\
\text { osteoporosis } \\
(\mathrm{n}=35 / 56)\end{array}$ & $\begin{array}{c}\text { Secondary } \\
\text { osteoporosis } \\
(\mathrm{n}=19 / 35)\end{array}$ \\
BP & 12 & 8 \\
VD & 2 & 4 \\
BP+VD & 5 & 1 \\
BP+VK & 0 & 1 \\
VD+VK & 1 & 0 \\
SERM & 4 & 1 \\
SERM+VD & 4 & 0 \\
TPTD & 7 & 4 \\
\hline
\end{tabular}

Values are presented as number of patients.

$\mathrm{BP}$, bisphosphonate; VD, activated vitamin D3; VK, vitamin K2; SERM, selective estrogen receptor modulator; TPTD, teriparatide.

(SAS Institute, Cary, NC, USA). For continuous variables, data are expressed as mean \pm standard deviation. Statistical differences between the groups were compared using paired or unpaired $t$-tests or the Mann-Whitney $U$ test, as appropriate. The chi-square test was used for categorical variables. Probability values less than 0.05 were considered statistically significant.

\section{Results}

\section{Comparison of background data and operative inva- siveness}

Background data of patients with primary osteoporosis and those with secondary osteoporosis are presented in Table 1. No significant differences in terms of age, gender, BMD of the proximal femur, or osteoporosis treatment prior to OVC were observed. Thirty-five patients (62.5\%) in the primary osteoporosis group and 19 (54.3\%) in the secondary osteoporosis group received anti-osteoporosis 
pharmacotherapy prior to sustaining OVC (Table 2). No significant difference in the use of anti-osteoporosis drugs was observed between the groups. Diabetes mellitus (51.4\%) was the most common cause of secondary osteoporosis, followed by glucocorticoid use (22.9\%) (Table 3).

The distribution of OVC is presented in Fig. 1. No significant difference in distribution was observed between the groups, with a peak observed in the region of the thoracolumbar junction in both groups. The comparison of operative invasiveness demonstrated no significant dif-

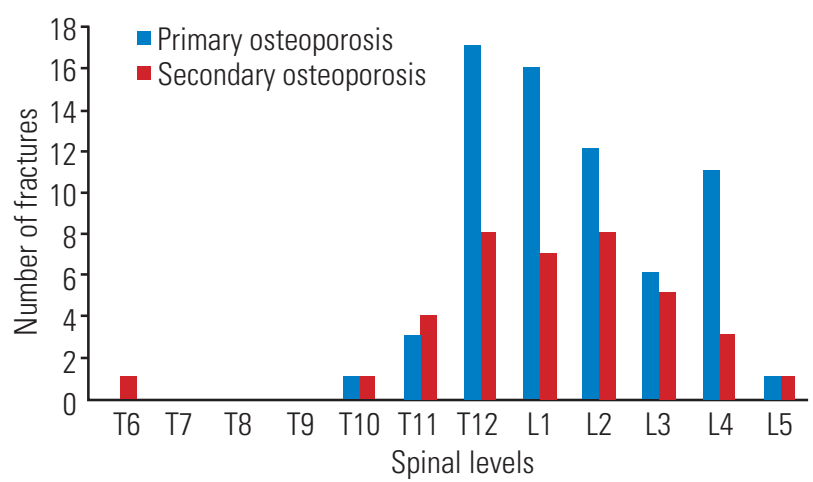

Fig. 1. Distribution of osteoporotic vertebral collapse (OVC) in the primary and secondary osteoporosis groups. Both groups had similar distributions of $\mathrm{OVC}$, with a peak in the region of the thoracolumbar junction.

Table 3. Cause of secondary osteoporosis (n=35)

\begin{tabular}{lc}
\hline Cause & No. of patients \\
\hline Diabetes mellitus & 18 \\
\hline Glucocorticoid use & 8 \\
\hline Excessive alcohol intake & 3 \\
\hline Post-gastrectomy & 3 \\
\hline Chronic renal failure & 2 \\
\hline Warfarin use & 1 \\
\hline
\end{tabular}

ferences between the groups in terms of surgery (PAVREC or $\mathrm{VP}+\mathrm{PSF}$ ), number of levels fused, operation time, estimated blood loss, or proportion of patients requiring transfusion (Table 4).

\section{Comparison of perioperative complications}

Nine patients (16.1\%) in the primary osteoporosis group and $8(22.9 \%)$ in the secondary osteoporosis group experienced perioperative complications. No significant difference in the incidence of perioperative complications was evident between the groups. Delirium, hematoma, urinary infection, pneumonia, and SSI were observed in both groups (Table 5).

SSI developed in 4 patients (11.4\%) in the secondary osteoporosis group and in 1 patient (1.8\%) in the primary osteoporosis group. The incidence of SSI was significantly higher in the secondary osteoporosis group $(p<0.05)$. No significant differences in the incidences of other perioperative complications were observed.

Among patients with SSI, deep SSI was seen in 2 patients in the secondary osteoporosis group (at 10 and 14 days after surgery) and 0 patients in the primary osteoporosis group. One patient with diabetes mellitus in the secondary osteoporosis group developed deep SSI and underwent open irrigation and debridement of infected tissues. Another patient in the secondary osteoporosis group with chronic renal failure developed methicillinresistant Staphylococcus aureus (MRSA) infection and ultimately underwent removal of the instrument. Patients with hematoma in both groups required open drainage; however, other patients in both groups with superficial SSI, delirium, decubitus ulcers, urinary infection, and pneumonia were successfully treated with conservative treatment. All superficial SSIs diagnosed at 7, 10, and 14

Table 4. Comparison of operative invasiveness between patients with primary and secondary osteoporosis

\begin{tabular}{|c|c|c|c|}
\hline Variables & Primary osteoporosis $(n=56)$ & Secondary osteoporosis $(n=35)$ & $p$-value \\
\hline Surgery (PAVREC:VP+PSF) & $42: 14$ & $21: 14$ & 0.132 \\
\hline No. of levels fused & $4.3 \pm 2.3$ & $4.2 \pm 2.6$ & 0.791 \\
\hline Operation time (min) & $281 \pm 77$ & $281 \pm 71$ & 0.970 \\
\hline Estimated blood loss (mL) & $851.6 \pm 777.5$ & $634.5 \pm 549.2$ & 0.153 \\
\hline Transfusion (yes:no) & $30: 26$ & $13: 22$ & 0.384 \\
\hline Perioperative complications (yes:no) & 9:47 & $8: 27$ & 0.419 \\
\hline
\end{tabular}

Values are presented as mean \pm standard deviation or number of patients.

PAVREC, posterior-approach vertebral replacement with rectangular parallelepiped cages; VP, vertebroplasty; PSF, posterior spinal fusion. 
Table 5. Perioperative complications occurred in patients with primary or secondary osteoporosis

\begin{tabular}{lcc} 
Complications & $\begin{array}{c}\text { Primary } \\
\text { osteoporosis } \\
(\mathrm{n}=9 / 56)\end{array}$ & $\begin{array}{r}\text { Secondary } \\
\text { osteoporosis } \\
(\mathrm{n}=8 / 35)\end{array}$ \\
Delirium & 3 & 1 \\
Hematoma & 2 & 1 \\
\hline Decubitus & 1 & 0 \\
Urinary infection & 1 & 1 \\
\hline Pneumonia & 1 & 1 \\
Surgical site infection & 1 & $4^{\text {a) }}$ \\
\hline
\end{tabular}

a)Including two cases with deep surgical site infection.

days after surgery were successfully treated with empirical antibiotic therapy.

\section{Discussion}

Progressive and symptomatic OVC should be surgically treated where possible to maintain the quality of life of patients. Indications for spinal instrumentation surgery in the elderly and patients with secondary osteoporosis should be carefully considered as these populations have a greater prevalence of comorbidities than patients with primary osteoporosis. However, to the best of our knowledge, no studies comparing patients with primary osteoporosis and those with secondary osteoporosis with regard to perioperative medical complications after spinal instrumentation surgery have been reported.

The present study was the first to directly compare perioperative medical complications after posterior approach spinal instrumentation surgery for OVC between patients with primary osteoporosis and those with secondary osteoporosis. The present study found no significant differences between the groups in terms of age, gender, BMD, osteoporosis treatment, OVC distribution, surgical procedure, or parameters for operative invasiveness. Postoperative SSI resulted from multiple patient- and procedure-related factors. Surgeons typically tend to select less invasive surgical procedures for patients with comorbidities, and this may reduce the incidence of complications. However, the results of the present study indicated that the incidences of perioperative medical complications are comparable between the primary and secondary osteoporosis groups under the same conditions of patient background and operative invasiveness.
In the present study, the incidence of perioperative medical complications did not significantly differ between the primary and secondary osteoporosis groups (16.1\% vs. $22.9 \%$ ); however, SSI was 6.3 times more common in the secondary osteoporosis group (11.4\%) than in the primary osteoporosis group $(1.8 \%$; $p<0.05)$. Furthermore, 2 patients in the secondary osteoporosis group, but none in the primary osteoporosis group, developed deep SSI requiring surgical intervention. In particular, 1 patient in the secondary osteoporosis group experienced severe deep SSI involving MRSA. These results suggest that the overall incidence of perioperative medical complications is similar between the primary and secondary osteoporosis groups under the same conditions of patient background and operative invasiveness; however, careful observations for the development of severe SSI should be performed in patients with secondary osteoporosis.

SSI should be avoided after spinal instrumentation surgery. In general, the presence of instruments increases the risk of SSI following spine surgery. The reported incidence of SSI following spinal instrumentation surgery (3.2\%7.2\%) [10-13] was higher than that following non-instrumented surgery $(0.9 \%-2.6 \%)[14,15]$. Established patientrelated risk factors for SSI include advanced age, obesity, diabetes mellitus, malnutrition, smoking, glucocorticoid use, and previous spinal surgery $[16,17]$. Osteoporosis is also considered to be a patient-related risk factor for spinal instrumented arthrodesis [17]. Schimmel et al. [8] reviewed 1,568 patients (1,615 procedures) who underwent lumbar or thoracolumbar spine fusion and compared 36 patients with deep SSI (2.2\%) after surgery with 135 randomly selected uninfected patients to identify risk factors for SSI. Diabetes mellitus was the most important patientrelated risk factor, with the risk of SSI almost six times higher than that in non-diabetic patients (odds ratio [OR], 5.92; 95\% confidence interval [CI], 1.23-28.5; $p=0.026$ ), followed by smoking habits (OR, 2.33; 95\% CI, 1.02-5.32; $p=0.045$ ) [8]. Diabetes and smoking are associated with tissue ischemia and small vessel damage, predisposing to an increased risk of SSI [16]. In the present study, diabetes mellitus was the most frequent cause of secondary osteoporosis.

Among comorbidities, kidney disease is considered the greatest risk factor for complications, including SSI. Puvanesarajah et al. [18] recently reported data from the Medicare database in the United States and found that among patients who underwent lumbar spinal fusion, the 
kidney disease cohort had increased rates of all medical complications compared to the control cohort comprising all other patients $(21.3 \%$ vs. $14.2 \%$; OR $1.64,95 \%$ CI $1.44-1.85, p<0.001$ ), with increased rates of infection also observed in the kidney disease cohort (4.4\% vs. $1.8 \%$; OR, 2.43; 95\% CI, 1.87-3.16; $p<0.001)$. Both 90 -day ( $1.1 \%$ vs. 0.2\%; OR, 5.05; 95\% CI, 2.90-8.77; $p<0.001)$ and 1-year (1.9\% vs. $0.7 \%$; OR, $2.77 ; 95 \%$ CI, $1.87-4.11 ; p<0.001)$ mortalities were significantly higher in the kidney disease cohort than in the control cohort [18]. According to $\mathrm{Pu}$ vanesarajah et al. and our experience in the present study of 1 patient with chronic renal failure who developed the most severe complication in our series of deep SSI with MRSA that required instrument removal, careful observation and strict anti-infection control are required to prevent SSI in patients with kidney disease.

Although the present study is, to our knowledge, the first to address the question of whether secondary osteoporosis is associated with more perioperative medical complications than primary osteoporosis after posterior approach spinal instrumentation surgery for OVC, several study limitations should be addressed. First, the present study was a retrospective study of a single cohort. Future prospective studies are required to determine the precise incidence of perioperative complications. Second, the present study focused only on medical complications; however, implant-related complications are also an important issue in patients with osteoporosis. The simultaneous assessment of medical and surgery-related complications for patients with primary osteoporosis and those with secondary osteoporosis should therefore be addressed in future studies. Third, the relatively small number of subjects compared to that previously reported epidemiological studies meant that we were unable to conduct multivariate analyses to evaluate the most significant factors affecting perioperative medical conditions in patients with primary osteoporosis and those with secondary osteoporosis. Further studies with larger populations are needed to verify our findings.

\section{Conclusions}

The overall incidence of perioperative medical complications after posterior approach spinal instrumentation surgery for OVC was comparable between the primary and secondary osteoporosis groups under the same conditions of patient background and operative invasiveness. How- ever, SSI (particularly severe cases) occurred more frequently in patients with secondary osteoporosis. Among causes of secondary osteoporosis, kidney disease may be a risk factor for severe SSI.

\section{Conflict of Interest}

No potential conflict of interest relevant to this article was reported.

\section{References}

1. Lehman RA Jr, Kang DG, Wagner SC. Management of osteoporosis in spine surgery. J Am Acad Orthop Surg 2015;23:253-63.

2. Kim KT, Suk KS, Kim JM, Lee SH. Delayed vertebral collapse with neurological deficits secondary to osteoporosis. Int Orthop 2003;27:65-9.

3. Mochida J, Toh E, Chiba M, Nishimura K. Treatment of osteoporotic late collapse of a vertebral body of thoracic and lumbar spine. J Spinal Disord 2001;14:393-8.

4. Choma TJ, Rechtine GR, McGuire RA Jr, Brodke DS. Treating the aging spine. J Am Acad Orthop Surg 2015;23:e91-100.

5. Hu SS, Berven SH. Preparing the adult deformity patient for spinal surgery. Spine (Phila Pa 1976) 2006;31(19 Suppl):S126-31.

6. Orimo H, Nakamura T, Hosoi T, et al. Japanese 2011 guidelines for prevention and treatment of osteoporosis: executive summary. Arch Osteoporos 2012;7:320.

7. Baron EM, Albert TJ. Medical complications of surgical treatment of adult spinal deformity and how to avoid them. Spine (Phila Pa 1976) 2006;31(19 Suppl):S106-18.

8. Schimmel JJ, Horsting PP, de Kleuver M, Wonders G, van Limbeek J. Risk factors for deep surgical site infections after spinal fusion. Eur Spine J 2010;19:17119.

9. Suzuki T, Abe E, Miyakoshi N, et al. Posterior-approach vertebral replacement with rectangular parallelepiped cages (PAVREC) for the treatment of osteoporotic vertebral collapse with neurological deficits. J Spinal Disord Tech 2013;26:E170-6.

10. Collins I, Wilson-MacDonald J, Chami G, et al. The diagnosis and management of infection following 
instrumented spinal fusion. Eur Spine J 2008;17:445450.

11. Mirovsky Y, Floman Y, Smorgick Y, et al. Management of deep wound infection after posterior lumbar interbody fusion with cages. J Spinal Disord Tech 2007;20:127-31.

12. Pappou IP, Papadopoulos EC, Sama AA, Girardi FP, Cammisa FP. Postoperative infections in interbody fusion for degenerative spinal disease. Clin Orthop Relat Res 2006;444:120-8.

13. Picada R, Winter RB, Lonstein JE, et al. Postoperative deep wound infection in adults after posterior lumbosacral spine fusion with instrumentation: incidence and management. J Spinal Disord 2000;13:425.

14. Friedman ND, Sexton DJ, Connelly SM, Kaye KS. Risk factors for surgical site infection complicating laminectomy. Infect Control Hosp Epidemiol 2007;28:1060-5.
15. Gaynes RP, Culver DH, Horan TC, Edwards JR, Richards C, Tolson JS. Surgical site infection (SSI) rates in the United States, 1992-1998: the National Nosocomial Infections Surveillance System basic SSI risk index. Clin Infect Dis 2001;33 Suppl 2:S69-77.

16. Chahoud J, Kanafani Z, Kanj SS. Surgical site infections following spine surgery: eliminating the controversies in the diagnosis. Front Med (Lausanne) 2014;1:7.

17. Koutsoumbelis S, Hughes AP, Girardi FP, et al. Risk factors for postoperative infection following posterior lumbar instrumented arthrodesis. J Bone Joint Surg Am 2011;93:1627-33.

18. Puvanesarajah V, Jain A, Hess DE, Shimer AL, Shen $\mathrm{FH}$, Hassanzadeh H. Complications and mortality after lumbar spinal fusion in elderly patients with late stage renal disease. Spine (Phila Pa 1976) 2016;41:E1298-302. 